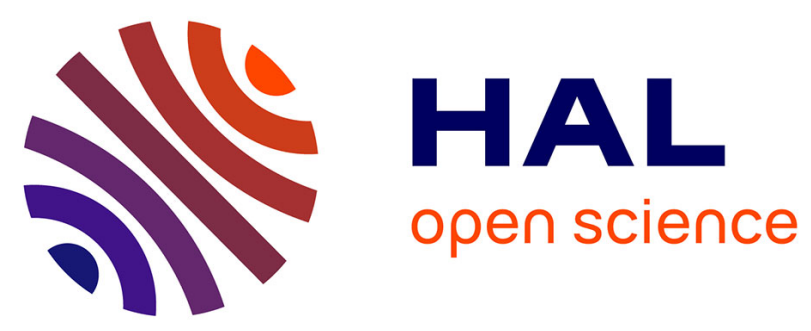

\title{
Long-Range Phase Coherence in Double-Barrier Magnetic Tunnel Junctions with a Large Thick Metallic Quantum Well
}

B. S. Tao, H. X. Yang, Y. L. Zuo, Xavier Devaux, Gwladys Lengaigne, Michel Hehn, Daniel Lacour, Stéphane Andrieu, M. Chshiev, Thomas Hauet, et al.

\section{To cite this version:}

B. S. Tao, H. X. Yang, Y. L. Zuo, Xavier Devaux, Gwladys Lengaigne, et al.. Long-Range Phase Coherence in Double-Barrier Magnetic Tunnel Junctions with a Large Thick Metallic Quantum Well. Physical Review Letters, 2015, 115 (15), pp.157204. 10.1103/PhysRevLett.115.157204 . hal-01284017

\section{HAL Id: hal-01284017 https://hal.science/hal-01284017}

Submitted on 13 Jul 2016

HAL is a multi-disciplinary open access archive for the deposit and dissemination of scientific research documents, whether they are published or not. The documents may come from teaching and research institutions in France or abroad, or from public or private research centers.
L'archive ouverte pluridisciplinaire HAL, est destinée au dépôt et à la diffusion de documents scientifiques de niveau recherche, publiés ou non, émanant des établissements d'enseignement et de recherche français ou étrangers, des laboratoires publics ou privés. 


\title{
Long-Range Phase Coherence in Double-Barrier Magnetic Tunnel Junctions with a Large Thick Metallic Quantum Well
}

\author{
B. S. Tao, ${ }^{1,2}$ H. X. Yang, ${ }^{3}$ Y. L. Zuo, ${ }^{2}$ X. Devaux, ${ }^{2}$ G. Lengaigne, ${ }^{2}$ M. Hehn, ${ }^{2}$ D. Lacour, ${ }^{2}$ S. Andrieu, ${ }^{2}$ M. Chshiev, ${ }^{3}$ \\ T. Hauet, ${ }^{2}$ F. Montaigne, ${ }^{2}$ S. Mangin, ${ }^{2}$ X. F. Han, ${ }^{1, \dagger}$ and Y. Lu ${ }^{2, *}$ \\ ${ }^{1}$ Beijing National Laboratory of Condensed Matter Physics, Institute of Physics, Chinese Academy of Sciences, Beijing 100190, China \\ ${ }^{2}$ Institut Jean Lamour, UMR 7198, CNRS-Université de Lorraine, BP239, 54506 Vandouvre-lès-Nancy, France \\ ${ }^{3}$ Univ. Grenoble Alpes, INAC-SPINTEC, F-38000 Grenoble, France; \\ CEA, INAC-SPINTEC, F-38000 Grenoble, France and CNRS, SPINTEC, F-38000 Grenoble, France
}

(Received 9 June 2015; published 9 October 2015)

\begin{abstract}
Double-barrier heterostructures are model systems for the study of electron tunneling and discrete energy levels in a quantum well (QW). Until now resonant tunneling phenomena in metallic QWs have been observed for limited thicknesses (1-2 nm) under which electron phase coherence is conserved. In the present study we show evidence of QW resonance states in Fe QWs up to $12 \mathrm{~nm}$ thick and at room temperature in fully epitaxial double $\mathrm{MgAlO}_{x}$ barrier magnetic tunnel junctions. The electron phase coherence displayed in this QW is of unprecedented quality because of a homogenous interface phase shift due to the small lattice mismatch at the $\mathrm{Fe}-\mathrm{MgAlO}_{x}$ interface. The physical understanding of the critical role of interface strain on QW phase coherence will greatly promote the development of spin-dependent quantum resonant tunneling applications.
\end{abstract}

DOI: 10.1103/PhysRevLett.115.157204

PACS numbers: $85.75 . \mathrm{Mm}, 72.25 . \mathrm{Ba}, 73.21 . \mathrm{Fg}$

Resonant tunneling in double-barrier quantum well (QW) structures has been extensively studied because of its importance in the field of nanoelectronic science and technology [1]. The double-barrier structure behaves as an optical interferometer. Thus, in order to observe electron resonant tunneling, the electron phase should be kept coherent when reflecting between the two potential barriers. Because of the loss of electron phase coherence, the resonant tunneling cannot survive if the two barriers are too far from each other. The decoherence process can be introduced by interface roughness [2], or inelastic scattering in the QW and at the interface [3], which can absorb and re-emit the electrons that lose their phase information.

Recently, the combination of the tunneling magnetoresistance (TMR) effect $[4,5]$ with resonant tunneling through metallic QW states [6,7] in magnetic tunnel junctions (MTJs) has triggered considerable interest in new functionality of spintronic devices operating in the quantum tunneling regime. In these structures, the QW potential barrier can be formed either by a metallic layer using the symmetry dependent band structure [8-13] or by double oxide tunneling barriers with a much greater barrier height for better electron confinement [14-16]. So far, to preserve good phase coherence, the metallic QW thickness in double-barrier magnetic tunnel junctions (DMTJs) has been limited to around 1-2 nm. In this case, it is impossible to modulate the Fermi level energy $E_{F}$ by making a direct electrical connection with the middle QW layer. To achieve good phase coherence and enhance the QW resonant tunneling effect with a large well thickness, the dephasing mechanisms involved in the QW and at the metal-oxide interface must be clarified.
In this study, we provide experimental evidence that these QW states can be greatly improved in fully epitaxial DMTJs based on a spinel $\mathrm{MgAlO}_{x}$ oxide barrier. This material was recently proposed as a promising barrier in MTJs [17-23] because of its nondeliquescence and small lattice mismatch with typical bcc ferromagnetic materials and Heusler alloys. Remarkably, up to ten separated QW resonance states were observed on large size patterned MTJs (100-900 $\left.\mu \mathrm{m}^{2}\right)$ with a $12 \mathrm{~nm}$ thick Fe QW. Moreover, we observed that the conductance oscillation amplitude in $\mathrm{MgAlO}_{x}$-based DMTJs is enhanced by almost 1 order of magnitude compared to MgO-based DMTJs with the same QW thickness. The unprecedented high quality of electron phase coherence in the $\mathrm{QW}$ with double $\mathrm{MgAlO}_{x}$ barriers was explained in terms of the strain related interface dephasing mechanism. Such peculiar transport properties give us the possibility to directly connect with the QW layer to independently control the energy of electrons that are injected into the QW as in a three-terminal device [24]. This added level of control could be used to generate new functionality in spin-dependent quantum resonant tunneling applications.

The epitaxial DMTJs with double $\mathrm{MgAlO}_{x}$ barriers were grown by molecular beam epitaxy (MBE). Figure 1 shows the schematic sample structure and band energy profile. The QW structure is formed in the middle $\mathrm{Fe}$ layer sandwiched by a thin (barrier I) and thick barrier (barrier II). Two series of samples were fabricated on $\mathrm{MgO}(001)$ substrates. The structures of the first series samples are $\mathrm{Fe}(45 \mathrm{~nm}) / \operatorname{MgAlO}_{x}(3 \mathrm{MLs}) / \mathrm{Fe}(t) / \operatorname{MgAlO}_{x}(12 \mathrm{MLs}) /$ $\mathrm{Fe}(10 \mathrm{~nm}) / \mathrm{Co}(20 \mathrm{~nm}) / \mathrm{Au}(15 \mathrm{~nm})$, where the thicknesses of the middle Fe layer were 6.3, 7.5, and 


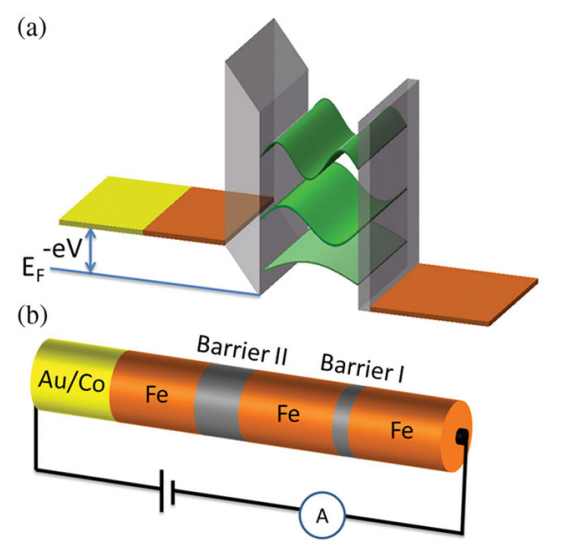

FIG. 1 (color online). (a) Energy profile in DMTJ structure and QW states at different energy levels. (b) Stack structure of DMTJ and setup of measurement.

$12.6 \mathrm{~nm}$, respectively. The second series samples were designed to investigate the effect of the barrier interface and the MTJ stacks are composed of $\mathrm{Fe}(45 \mathrm{~nm}) / \mathrm{barrier}$ I (3 MLs)/Fe(10 nm)/barrier II (12 MLs)/Fe(10 nm)/ $\mathrm{Co}(20 \mathrm{~nm}) / \mathrm{Au}(15 \mathrm{~nm})$, where both barrier I and II can be either $\mathrm{MgO}$ or $\mathrm{MgAlO}_{x}$. All numbers represent thicknesses in nanometers and ML stands for atomic monolayer. Please see the Supplemental Material for details concerning growth and sample structural characterization [25].

First, let us evaluate the magnetotransport properties in one $\mathrm{MgAlO}_{x}$ DMTJ with QW thickness $t=7.5 \mathrm{~nm}$. The inset of Fig. 2(a) shows the representative TMR curve measured at different temperatures from 16 to $295 \mathrm{~K}$. The magnetic field $H$ was applied along the $\mathrm{Fe}[100]$ easy axis direction and the TMR ratio is calculated as $\left(R_{\mathrm{AP}}-R_{P}\right) / R_{P} \times 100 \%$, where $R_{\mathrm{AP}}$ and $R_{P}$ are the resistances of the antiparallel (AP) and parallel $(\mathrm{P})$ magnetization configurations, respectively. From the shape of the TMR curves, it is possible to identify that the hard layer is the top Fe-Co layer and the free layer is composed of the middle and bottom Fe layers, which are ferromagnetically coupled through the $3 \mathrm{ML}$ thin barrier (see more magnetic characterizations in the Supplemental Material [25]). Figures 2(a)-2(b) show the differential conductance $(d I / d V)$ curves measured at different temperature in the $\mathrm{P}$ and AP states, which are normalized with the conductance $G_{\mathrm{P}}(0 \mathrm{~V}, 16 \mathrm{~K})$. In the $\mathrm{P}$ state, some strong oscillations of the conductance are observed in the negative bias region where the electrons are injected from top electrodes into the QW. The separation of the local maximum peak gradually increases at higher negative bias. Although the oscillation amplitude attenuates with increasing temperature, the oscillatory feature is still observable even at RT with the periodicity remaining almost unchanged. Another interesting aspect is that the oscillatory feature is still observable in the AP state although with a significantly reduced amplitude. The periodicity and maximum peak positions are almost the same as those in the P state, which was not observed in MgO DMTJs [14,15]. In Fig. 2(c), the differential tunneling magnetoresistance
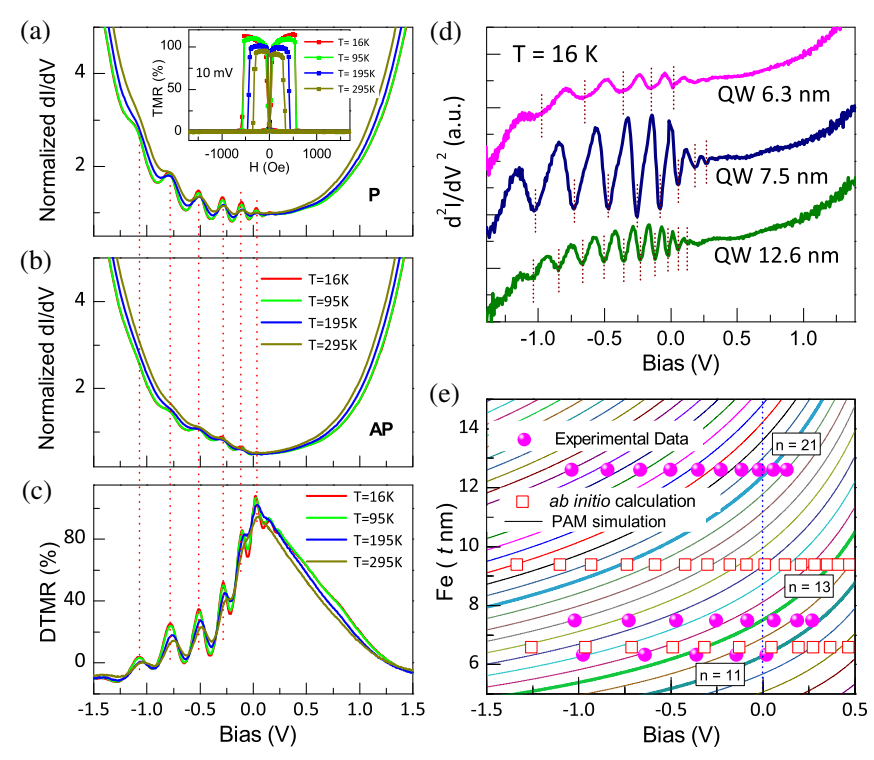

FIG. 2 (color online). Normalized conductance as a function of bias voltage in the (a) P and (b) AP state, respectively. (c) DTMR dependence with bias voltage. (d) QW thickness dependence of $d^{2} I / d V^{2}$ curves in the $\mathrm{P}$ state (measured at $16 \mathrm{~K}$ ). The dashed lines indicate the resonant peak positions. (e) QW peak positions for the experimental results (circles), PAM simulation results (lines), and $a b$ initio calculation results (squares). The three numbers $n$ in the figure represent the QW node number just below $E_{F}$ for the three samples with different QW thickness.

(DTMR) curves calculated from the differential $d I / d V$ curves in the P and AP states also show clear oscillations with the same phases as those of the conductivity oscillations.

In order to confirm that the observed conductance oscillations originate from the QW states in the middle Fe layer, the bias dependent conductance was measured for two other $\mathrm{MgAlO}_{x}$ DMTJs with a different middle Fe thickness: 6.3 and $12.6 \mathrm{~nm}$. To precisely measure the $\mathrm{QW}$ energy position, the secondary differential $d^{2} I / d V^{2}$ curves were deduced from the normalized $d I / d V$ curves in the $\mathrm{P}$ state, which are plotted in Fig. 2(d). The QW energy position is defined at the local minimum of the $d^{2} I / d V^{2}$ curves, as marked with the dashed lines. All samples show clear oscillation behaviors with different amplitudes and periodicity. The increase of Fe thickness results in a shorter periodicity, which proves that the observed oscillations are coming from the QW states in the middle Fe layer. The highest oscillation amplitude was observed for the Fe QW thickness $t=7.5 \mathrm{~nm}$. These oscillations are surprising in their clarity and number (up to 10) and are still observable for DMTJ with $t=12.6 \mathrm{~nm}$. Since the QW state is formed in the Fe majority $\Delta_{1}$ band $[7,14]$, the decrease of the oscillation amplitude in the $t=$ $12.6 \mathrm{~nm}$ sample can be understood as being due to the finite mean free path of the majority $\Delta_{1}$ electron for conserving its energy, symmetry, and phase information. A well-defined quantum-statistical calculation [2,32] has shown that the QW states are quenched far before the QW thickness reaches the distance of the mean free path. Therefore, we can conclude 
that the mean free path as well as the phase coherence length in our Fe QW should be much longer than $12 \mathrm{~nm}$ and the reported values [33-35]. To precisely determine this phase coherence length, $\mathrm{MgAlO}_{x}$ DMTJs with thicker QWs are needed. This is the first time that the $\mathrm{QW}$ resonant tunneling phenomenon has been observed in metallic QW devices of large thicknesses, at least greater than $10 \mathrm{~nm}$. We believe this is reasonable because QW states have been observed by angle-resolved photoemission measurements in quite thick Ag layers, up to 112 monolayers (24.3 nm) on Fe (100) [36]. As for the sample with $t=6.3 \mathrm{~nm}$, the smaller amplitude could be due to the increase of the middle Fe roughness after annealing due to the poor wetting property of the thin metal film on the oxide. (See more information about the three samples in the Supplemental Material [25].)

The QW states obtained from the experimental results can be directly and qualitatively compared to a simple phase accumulation model (PAM) [37]. The PAM describes the quantization condition for the existence of a $\mathrm{QW}$ state as

$$
2 k_{\perp} d-\Phi_{1}-\Phi_{2}-\Phi_{\mathrm{inf}}=2 \pi n
$$

where $k_{\perp}=\sqrt{2 m^{*}\left(E-E_{L}\right)} / \hbar$ is the crystal momentum wave vector in the film perpendicular to the interface, $d$ is the $\mathrm{Fe}$ QW thickness, and $\Phi_{1}=\Phi_{2}=$ $2 \sin ^{-1} \sqrt{\left(E-E_{L}\right) /\left(E_{U}-E_{L}\right)}-\pi$ is the reflection phase shift at the two $\mathrm{Fe}-\mathrm{MgAlO}_{x}$ interfaces. Furthermore, $m^{*}$ is the effective mass of the majority $\Delta_{1}$ electron in $\mathrm{Fe}$, and $E_{L}$ and $E_{U}$ are the energies of the lower and upper edges of the barrier band gap. Here, we set $E_{L}=-1.0 \mathrm{eV}$ and $E_{U}=3.9 \mathrm{eV}$, similarly to Ref. [7]. An important parameter $\Phi_{\text {inf }}$ is taken into account for the additional phase shift at interfaces due to other effects such as interface roughness, chemical disorder, impurities, and strain inhomogeneity, etc. To qualitatively compare our results with such an analysis, we first set $\Phi_{\text {inf }}=0$, which will be discussed below. As displayed in Fig. 2(e) in the solid lines, the simulated results show fairly good agreement with the experimental results when choosing $m^{*}=1$. It is found that the PAM can qualitatively reproduce the QW positions for samples with 6.3 and $7.5 \mathrm{~nm} \mathrm{Fe}$. For $12.6 \mathrm{~nm} \mathrm{Fe}$, larger error develops at higher bias. To further quantitatively determine the QW position with such a thick Fe layer, ab initio calculations were performed to calculate the $s$-resolved partial DOS at the $\bar{\Gamma}$ point within the two central $\mathrm{Fe}$ layers in the bcc Fe $\left|[\mathrm{MgO}]_{7}\right| \mathrm{Fe} \mid[\mathrm{MgO}]_{7}$ structure (see the Supplemental Material for details [25]). In the calculations, structures with two different $\mathrm{Fe}$ thicknesses equal to $67 \mathrm{MLs}(9.4 \mathrm{~nm})$ and $47 \mathrm{MLs}(6.5 \mathrm{~nm})$ were used and $\mathrm{MgAl}_{2} \mathrm{O}_{4}$ were replaced by 7 MLs of MgO to simplify the calculation load. The QW peak positions derived from the sharp majority DOS spikes of the middle Fe film are marked in Fig. 2(e) with open squares. The calculated QW states are in very good agreement with the PAM simulations as well as with the experimental results, which further confirms that the observed oscillation in the rather thick Fe layer originates from the QW resonant states.

The observed long-range phase coherence in such a thick Fe QW in our $\mathrm{MgAlO}_{x}$ DMTJs could be due to the better quality of the $\mathrm{Fe} \mathrm{QW}, \mathrm{Fe}-\mathrm{MgAlO}_{x}$ interface or the $\mathrm{MgAlO}_{x}$ barrier itself. To elucidate the origin, four DMTJ samples were prepared with different configurations of two types of barriers: $\mathrm{MgO}$ and $\mathrm{MgAlO}_{x}$, as listed in Table I. Figures 3(a) and 3(b) show the normalized $d^{2} I / d V^{2}$ curves in the $\mathrm{P}$ and AP states for two samples with a thin $\mathrm{MgO}$ barrier ( $B$ and $D$ ), respectively. [Samples $A$ and $C$ are shown in Figs. S7(a) and S7(c), respectively, of the Supplemental Material [25].] A clear feature is that the samples with thick $\mathrm{MgAlO}_{x}$ barriers $(C$ and $D)$ have an almost 1 order of magnitude stronger for the oscillation amplitude in the $\mathrm{P}$ state than those with thick $\mathrm{MgO}$ barriers $(A$ and $B)$. It seems that the bottom thin barrier has no influence on the oscillation amplitude, regardless of whether it is $\mathrm{MgO}$ or $\mathrm{MgAlO}_{x}$. In the AP state, no oscillatory feature can be observed for the samples with a thick $\mathrm{MgO}$ barrier $(A$ and $B$ ). The two peaks at -0.2 and $-1.0 \mathrm{eV}$ are related to the interface resonant state at the high quality Fe-MgO interface [38]. However, for samples
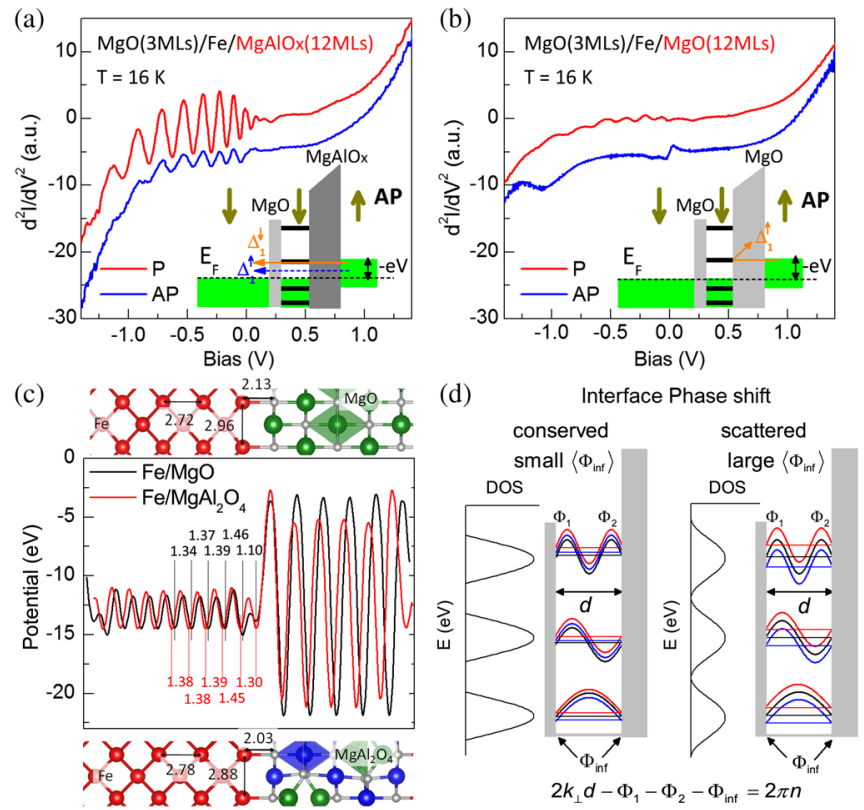

FIG. 3 (color online). $d^{2} I / d V^{2}$ curves in the $\mathrm{P}$ and AP states for DMTJs with (a) $\mathrm{MgO}(3 \mathrm{MLs}) / \mathrm{Fe} / \mathrm{MgAlO}_{x}(12 \mathrm{MLs})$ and (b) $\mathrm{MgO}(3 \mathrm{MLs}) / \mathrm{Fe} / \mathrm{MgO}(12 \mathrm{MLs})$ structures, respectively. Insets: schematics of tunneling of electrons with $\Delta_{1}$ symmetry in the AP configuration in samples with a thick $\mathrm{MgAlO}_{x}$ and $\mathrm{MgO}$ barrier, respectively. (c) Electrostatic potentials and structures of $\mathrm{Fe}-\mathrm{MgO}$ and $\mathrm{Fe}-\mathrm{MgAl}_{2} \mathrm{O}_{4}$ layers. The width $(\AA)$ between potential valleys in the $\mathrm{Fe}$ layers is shown for the $\mathrm{Fe}-\mathrm{MgO}$ (black) and $\mathrm{Fe}-\mathrm{MgAl}_{2} \mathrm{O}_{4}$ (red) interface. The lattice constant and length of the $\mathrm{Fe}-\mathrm{O}$ bond are also shown in the structure. (d) Schematics of the DOS in the QW with a large and small interface phase shift distribution. $\Phi_{1}, \Phi_{2}$ represent the phase shift on reflection at the interface and $\Phi_{\text {inf }}$ stands for the interface phase shift. 
with a thick $\mathrm{MgAlO}_{x}$ barrier $(C$ and $D)$, clear but attenuated oscillation is still maintained in the AP state with the same periodicity as in the $\mathrm{P}$ state. This can be understood by the band folding effect in the spinel $\mathrm{MgAl}_{2} \mathrm{O}_{4}$ MTJ, as demonstrated by the $a b$ initio calculations [23]. This band folding effect induces a coupling of the $\Delta_{1}$ evanescent state inside the barrier with the minority-spin state in the Fe electrode, which enhances the $\Delta_{1}$ conductance in the AP state and results in the observed resonant tunneling oscillation. We schematically illustrate this mechanism in the insets of Figs. 3(a) and 3(b) by taking into account only the $\Delta_{1}$ conductance. When the band folding effect is present, the additional $\Delta_{1}$ conductance in the AP state will also limit the TMR ratio $[18,23]$. As exactly found in Table I, the TMR ratios in the DMTJs with thick $\mathrm{MgAlO}_{x}$ barriers $(<200 \%$ at $16 \mathrm{~K})$ are lower than those with thick $\mathrm{MgO}$ barriers $(\sim 300 \%$ at $16 \mathrm{~K})$.

Since the samples have the same bottom stack layers except for the thick barriers (for $A$ and $C$ or for $B$ and $D$ ), the quality of their Fe QWs should be identical. This excludes the possibility of a different QW quality as the reason for the different oscillation amplitude. In addition, the contribution of the majority $\Delta_{1}$ channel in the total parallel conductance $G_{\Delta 1}^{\uparrow \uparrow} / G_{\mathrm{P}}$ was examined to determine if the symmetry filtering effect of the barrier [39] could play a role. However, the slight difference of $G_{\Delta 1}^{\uparrow \uparrow} / G_{\mathrm{P}}$ in both types of barriers estimated from their TMR ratio cannot explain the 1 order higher oscillation amplitude in the $\mathrm{MgAlO}_{x}$ DMTJs (see the Supplemental Material for details [25]). Finally, all evidence points to the Fe-thick barrier interface. To elucidate the interface related mechanisms, we have calculated electrostatic potential profiles for two structures: $\mathrm{Fe}_{11} \mid[\mathrm{MgO}]_{5}$ and $\mathrm{Fe}_{11} \mid\left[\mathrm{MgAl}_{2} \mathrm{O}_{4}\right]$, as shown in Fig. 3(c). Since the wave function decays exponentially inside the barrier region, sufficient barrier height is important to confine the QW states. The first requirement is to check the barrier heights for both $\mathrm{MgO}$ and $\mathrm{MgAl}_{2} \mathrm{O}_{4}$. It is found that the bond of $\mathrm{Fe}-\mathrm{O}$ is stronger at the $\mathrm{Fe}-\mathrm{MgAl}_{2} \mathrm{O}_{4}$ interface $(\mathrm{Fe}-\mathrm{O}$ distance $2.03 \AA$ ) than at the $\mathrm{Fe}-\mathrm{MgO}$ interface $(2.13 \AA)$. This induces a slightly higher potential barrier at the $\mathrm{Fe}-\mathrm{MgAl}_{2} \mathrm{O}_{4}$ interface. However, the potential barrier decreases within the insulator. Therefore, the $\mathrm{Fe}-\mathrm{MgAl}_{2} \mathrm{O}_{4}$ interface has no advantage from the comparison of the barrier height. Next, the lattice constant of $\mathrm{Fe}$ is examined. Because of the large lattice mismatch

TABLE I. Summary of experimental results for samples with different barrier configurations.

\begin{tabular}{|c|c|c|c|c|c|c|c|}
\hline \multirow[b]{2}{*}{ Sample } & \multirow{2}{*}{$\begin{array}{c}\text { Barrier } \\
\text { I } \\
\text { (3 MLs) }\end{array}$} & \multirow{2}{*}{$\begin{array}{c}\text { Barrier } \\
\text { II } \\
\text { (12 MLs) }\end{array}$} & \multicolumn{2}{|c|}{$\begin{array}{c}\text { TMR } \\
(10 \mathrm{mV})\end{array}$} & \multicolumn{2}{|c|}{$\begin{array}{l}\text { Oscillation } \\
\text { amplitude } \\
\text { (a.u.) }\end{array}$} & \multirow{2}{*}{$\begin{array}{c}\mathrm{Fe} \\
\mathrm{QW} \\
t(\mathrm{~nm})\end{array}$} \\
\hline & & & $T=295 \mathrm{~K}$ & $T=16 \mathrm{~K}$ & $\mathrm{P}$ & AP & \\
\hline $\bar{A}$ & $\operatorname{MgAlO}_{x}$ & $\mathrm{MgO}$ & $192 \%$ & $297 \%$ & 0.86 & $\ldots$ & 9.8 \\
\hline$B$ & $\mathrm{MgO}^{\wedge}$ & $\mathrm{MgO}$ & $176 \%$ & $300 \%$ & 0.71 & $\ldots$ & 9.6 \\
\hline$C$ & $\operatorname{MgAlO}_{x}$ & $\mathrm{MgAlO}_{x}$ & $130 \%$ & $177 \%$ & 6.55 & 1.72 & 12.6 \\
\hline$D$ & $\mathrm{MgO}$ & $\mathrm{MgAlO}_{x}$ & $121 \%$ & $198 \%$ & 10.17 & 2.42 & 10.0 \\
\hline
\end{tabular}

between $\mathrm{Fe}$ and $\mathrm{MgO}$, it is clear that the $\mathrm{Fe}$ is under a tensile strain at the interface with $\mathrm{MgO}$ since the lattice constant is $2.96 \AA$ in plane and $2.72 \AA$ out of plane. For the $\mathrm{MgAl}_{2} \mathrm{O}_{4}$ case, the strain in $\mathrm{Fe}$ is much smaller with a lattice constant of $2.88 \AA$ in plane and $2.78 \AA$ out of plane. Because of the interface strain, the period of the electrostatic potential in $\mathrm{Fe}$ is changed. It can be seen in Fig. 3(c) that in the case of the $\mathrm{Fe}-\mathrm{MgO}$ interface, the variation of the potential valley width for the interfacial $\mathrm{Fe}$ layers is much stronger (from 1.10 to $1.46 \AA$ ) compared to that at the $\mathrm{Fe}-\mathrm{MgAl}_{2} \mathrm{O}_{4}$ interface (from 1.30 to $1.45 \AA$ ). This condition is even valid farther from the interface, where the $\mathrm{Fe}$ potential valley width varies from 1.39 to $1.34 \AA$ in the $\mathrm{Fe}-\mathrm{MgO}$ case while varying only from 1.39 to $1.38 \AA$ in the $\mathrm{Fe}-\mathrm{MgAl}_{2} \mathrm{O}_{4}$ case. These irregular potential period changes at the $\mathrm{Fe}-\mathrm{MgO}$ interface will undoubtedly result in a significant interface phase shift $\Phi_{\text {inf }}$ for the QW states, as illustrated in Eq. (1). However, since the collected current is from a two-dimensional spatial integration in the whole junction area, if the electron changes its phase with the same $\Phi_{\text {inf }}$ everywhere, strong QW oscillations can still be obtained but with a shift of energy positions. Therefore, other mechanisms should exist to induce a large distribution of $\Phi_{\text {inf }}$, causing the vanishing of the QW oscillation. As schematically shown in Fig. 3(d), with a large distribution of $\left\langle\Phi_{\text {inf }}>\right.$, the QW energy position will also have a large distribution within the same QW index at different spatial locations. As a consequence, this smears the contrast of the current intensity as a function of bias and gives rise to the decrease of $\mathrm{QW}$ oscillation amplitude.

The mechanism introducing a large $\Phi_{\text {inf }}$ distribution can be highlighted by the creation of a misfit dislocation due to the lattice mismatch induced interface strain. For the $\mathrm{Fe}-\mathrm{MgO}$ case, when the thickness of $\mathrm{MgO}$ goes beyond 5 MLs [40], 1/2 $\langle 011\rangle$ misfit dislocations occur to relax the $\mathrm{MgO}$ lattice, which can then propagate to the bottom interface. This is observed by noting the appearance of a V-shaped feature [41-43] in the RHEED pattern [Fig. S1(b) of the Supplemental Material [25]]. This propagation will result in a long-range stress field around the core dislocations [44], leading to a significant interatomic distance distribution in the plane of the interface. Such a distribution generates an important phase shift scattering by varying the potential period as shown in the ab initio calculations and consequently leads to a large $\Phi_{\mathrm{inf}}$ spreading. The average distance $L$ between the adjacent misfit dislocations can be estimated to be $6 \mathrm{~nm}$ for $\mathrm{MgO}$ and $90 \mathrm{~nm}$ for $\mathrm{MgAl}_{2} \mathrm{O}_{4}$ from a crude static model [44]: $L=a / 2 f$ where $a$ is the lattice constant of the oxide and $f$ indicates the mismatch with $\mathrm{Fe}$. It is important to note that at the Fe-thin oxide ( $3 \mathrm{MLs}$ ) interface, the lattice of $\mathrm{MgO}$ is unrelaxed without misfit dislocation creation as confirmed by the RHEED pattern [Fig. S1(a) of the Supplemental Material [25] ]. No important difference in the conductivity oscillation amplitude is found when the 
bottom thin barrier (3 MLs) is $\mathrm{MgO}$ or $\mathrm{MgAlO}_{x}$. For the 12 ML thick $\mathrm{MgAlO}_{x}$ layer on $\mathrm{Fe}$, the small lattice mismatch induced strain creates very few misfit dislocations. In the RHEED patterns [Fig. S1(b) of the Supplemental Material [25] ], the dislocation related $\mathrm{V}$ shape feature is not observed. Furthermore, the persistence of Kikuchi lines signifies a good crystalline coherence in the whole barrier. Finally, the elimination of misfit dislocations conserves a homogenous and small distribution of $\Phi_{\mathrm{inf}}$, and in turn results in a significant enhancement of the QW conductivity oscillation.

In summary, the spin-dependent resonant tunneling properties in fully epitaxial $\mathrm{MgAlO}_{x}$ DMTJs grown by MBE have been studied. QW states in conductance curves as a function of the bias voltage are evidenced for up to a $12 \mathrm{~nm}$ thick Fe QW layer. Both PAM simulations and first principle calculations agree well with experimental results. Comparing experimental results using either $\mathrm{MgO}$ or $\mathrm{MgAlO}_{x}$ insulating barriers in these DMTJs allows us to highlight the key role of misfit dislocations in the barriers for the QW state establishment. Significant enhancement of the amplitude of the conductivity oscillation is observed up to 1 order of magnitude in the $\mathrm{MgAlO}_{x}$ DMTJs. This illustrates that the control of interface strain is essential to the preservation of a homogenous interface phase shift in order to obtain a sizable QW resonant tunneling oscillation.

This work is supported by the joint French National Research Agency (ANR)-National Science Foundation of China (NSFC) SISTER project (Grants No. ANR-11-IS100001 and No. NNSFC 61161130527) and ENSEMBLE project (Grants No. ANR-14-0028-01 and No. NNSFC 61411136001) as well as by Région Lorraine. X.F.H. acknowledges support from Ministry of Science and Technology (MOST) Projects No. 2010CB934401 and No. 2011YQ120053 and from National Natural Science Foundation (NSFC) Grant No. 11434014.

* Corresponding author. yuan.lu@univ-lorraine.fr Corresponding author. xfhan@iphy.ac.cn

[1] R. Tsu and L. Esaki, Appl. Phys. Lett. 22, 562 (1973).

[2] M. Chshiev, D. Stoeffler, A. Vedyayev, and K. Ounadjela, J. Magn. Magn. Mater. 240, 146 (2002).

[3] M. Jonson and A. Grincwajg, Appl. Phys. Lett. 51, 1729 (1987).

[4] M. Jullière, Phys. Lett. 54A, 225 (1975).

[5] J. S. Moodera, L. R. Kinder, T. M. Wong, and R. Meservey, Phys. Rev. Lett. 74, 3273 (1995).

[6] Z. Y. Lu, X.-G. Zhang, and S. T. Pantelides, Phys. Rev. Lett. 94, 207210 (2005).

[7] Y. Wang, Z. Y. Lu, X.-G. Zhang, and X. F. Han, Phys. Rev. Lett. 97, 087210 (2006).
[8] S. Yuasa, T. Nagahama, and Y. Suzuki, Science 297, 234 (2002).

[9] T. Nagahama, S. Yuasa, Y. Suzuki, and E. Tamura, J. Appl. Phys. 91, 7035 (2002).

[10] F. Greullet, C. Tiusan, F. Montaigne, M. Hehn, D. Halley, O. Bengone, M. Bowen, and W. Weber, Phys. Rev. Lett. 99, 187202 (2007).

[11] T. Niizeki, N. Tezuka, and K. Inomata, Phys. Rev. Lett. 100, 047207 (2008).

[12] P. Sheng, F. Bonell, S. Miwa, T. Nakamura, Y. Shiota, S. Murakami, D. D. Lam, S. Yoshida, and Y. Suzuki, Appl. Phys. Lett. 102, 032406 (2013).

[13] J. M. Teixeira, J. D. Costa, J. Ventura, J. B. Sousa, P. Wisniowski, and P. P. Freitas, Appl. Phys. Lett. 104, 112414 (2014).

[14] T. Nozaki, N. Tezuka, and K. Inomata, Phys. Rev. Lett. 96,027208 (2006).

[15] R. S. Liu et al., Phys. Rev. B 87, 024411 (2013).

[16] A. Iovan, S. Andersson, Yu. G. Naidyuk, A. Vedyaev, B. Dieny, and V. Korenivski, Nano Lett. 8, 805 (2008).

[17] H. Sukegawa, H. Xiu, T. Ohkubo, T. Furubayashi, T. Niizeki, W. Wang, S. Kasai, S. Mitani, K. Inomata, and K. Hono, Appl. Phys. Lett. 96, 212505 (2010).

[18] H. Sukegawa, Y. Miura, S. Muramoto, S. Mitani, T. Niizeki, T. Ohkubo, K. Abe, M. Shirai, K. Inomata, and K. Hono, Phys. Rev. B 86, 184401 (2012).

[19] H. Sukegawa, S. Mitani, T. Ohkubo, K. Inomata, and K. Hono, Appl. Phys. Lett. 103, 142409 (2013).

[20] B. S. Tao, D. Li, H. Liu, H. Wei, J.-F. Feng, S. Wang, and X. Han, IEEE Trans. Magn. 50, 4401004 (2014).

[21] B. S. Tao et al., Appl. Phys. Lett. 105, 102407 (2014).

[22] J. Zhang, X.-G. Zhang, and X. F. Han. Appl. Phys. Lett. 100, 222401 (2012).

[23] Y. Miura, S. Muramoto, K. Abe, and M. Shirai, Phys. Rev. B 86, 024426 (2012).

[24] M. Hehn, F. Montaigne, and A. Schuhl, Phys. Rev. B 66, 144411 (2002).

[25] See Supplemental Material at http://link.aps.org/ supplemental/10.1103/PhysRevLett.115.157204, which includes Refs. [26-31]. In this material, the methods, structural characterization, QW thickness determination, magnetic properties of DMTJs, and the effects from Fe thickness or barrier interface are described.

[26] G. Kresse and J. Hafner, Phys. Rev. B 47, 558 (1993).

[27] G. Kresse and J. Furthmüller, Phys. Rev. B 54, 11169 (1996).

[28] Y. Wang and J. P. Perdew, Phys. Rev. B 44, 13298 (1991).

[29] G. Kresse and D. Joubert, Phys. Rev. B 59, 1758 (1999).

[30] H. X. Yang, M. Chshiev, A. Kalitsov, A. Schuhl, and W. H. Butler, Appl. Phys. Lett. 96, 262509 (2010).

[31] A. M. Ceschin and J. Massies, J. Cryst. Growth 114, 693 (1991).

[32] M. Chshiev, D. Stoeffler, A. Vedyayev, and K. Ounadjela, Europhys. Lett. 58, 257 (2002).

[33] B. A. Gurney, V. S. Speriosu, J.-P. Nozieres, H. Lefakis, D. R. Wilhoit, and O. U. Need, Phys. Rev. Lett. 71, 4023 (1993).

[34] S. van Dijken, X. Jiang, and S. S. P. Parkin, Phys. Rev. B 66, 094417 (2002).

[35] D. P. Pappas, K.-P. Kämper, B. Miller, H. Hopster, D. Fowler, C. Brundle, A. Luntz, and Z.-X. Shen, Phys. Rev. Lett. 66, 504 (1991).

[36] J. J. Paggel, T. Miller, and T.-C. Chiang, Science 283, 1709 (1999). 
[37] N. V. Smith, N. B. Brookes, Y. Chang, and P. D. Johnson, Phys. Rev. B 49, 332 (1994).

[38] Y. Lu et al., Phys. Rev. B 86, 184420 (2012).

[39] W. H. Butler, X.-G. Zhang, T. C. Schulthess, and J. M. MacLaren, Phys. Rev. B 63, 054416 (2001).

[40] J. L. Vassent, M. Dynna, A. Marty, B. Gilles, and G. Patrat, J. Appl. Phys. 80, 5727 (1996).

[41] F. Bonell, Ph.D. thesis, Université de Lorraine, 2009.
[42] M. Dynna, J. L. Vassent, A. Marty, and B. Gilles, J. Appl. Phys. 80, 2650 (1996).

[43] M. Klaua, D. Ullmann, J. Barthel, W. Wulfhekel, J. Kirschner, R. Urban, T. L. Monchesky, A. Enders, J. F. Cochran, and B. Heinrich, Phys. Rev. B 64, 134411 (2001).

[44] F. Bonell, S. Andrieu, C. Tiusan, F. Montaigne, E. Snoeck, B. Belhadji, L. Calmels, F. Bertran, P. Le Fèvre, and A. Taleb-Ibrahimi, Phys. Rev. B 82, 092405 (2010). 